\title{
Cloud Based Remote FPGA Lab Platform: An Application of Internet of Things
}

\author{
SYED ABBAS ALI*, RAHEELA ASIF*, SAMAN HINA*, AND ZAINAB FATIMA*
}

RECEIVED ON 04.07.2017 ACCEPTED ON 13.11.2017

\begin{abstract}
IoT (Internet of Things) is the next generation of the Internet. The main goal of IoT is to connect each and every physical object to the Internet Cloud. This concept is introduced by bringing IoT technology to the laboratories, making expensive laboratory equipment available on-cloud for real-time experimentation. In this paper, an on CLP(Cloud Laboratory Platform) is presented by employing the concept of IoT to the academic experimentation environment. The CLP allows a rapid deployment of an online laboratory system enabling students and researchers to perform actual experiments on the on-Cloud laboratory equipment using a web interface. A web interface for end users to access front end of the system. This interface was developed for login purposes so that any user can perform experiments from anywhere. The interface also provides options for comments and feedback. Moreover, this research contribution also facilitate users to test their designs and record observations in real-time on the equipment. For demonstration purposes, a remote lab has been developed for high-tech Xilinx FPGA (Field Programmable Gate Array) development boards, namely Spartan-II and Spartan-III. This project aims to provide students a new tool to enhance their learning experience and encourage them to test their theoretical knowledge in practical applications.
\end{abstract}

Key Words: Cloud, Online Test-Beds, Internet of Things, Field Programmable Gate Array, Very Large Scale Integration, Remote Lab.

\section{INTRODUCTION}

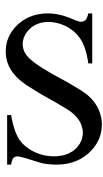

ome of the educational institutions of Pakistan have a problem with overcrowding. Many students have to perform laboratory experiments at the same time on the same piece of equipment. This often leads to most of them left with little understanding of how the instruments are being operated and a handful which have the know-how, but not the practical experience. This results in them not being interested in the practical applications of what they have learnt in class, thus not producing any sort of meaningful output. This issue is unavoidable as many institutions are not properly funded or staffed hence they do not have the necessary resources to teach each and every student.

To counteract this problem, many solutions exist; for instance, simulation software and virtual instruments.

Authors E-Mail: (saaj@neduet.edu.pk, engr_raheela@yahoo.com, samhaque@neduet.edu.pk, saaj.research@hotmail.com)

*Department of Computer \& Information Systems Engineering, NED University of Engineering \& Technology, Karachi

This is an open access article published by Mehran University Research Journal of Engineering and Technology, Jamshoro under the CC by 4.0 International License. 
In these kinds of scenarios, the problems of simulation complexity and behavioral model accuracy always exist. Another solution is to create remote laboratories or test-beds. This solution is more beneficial and is adopted by many institutions and universities all over the world [1].

A remote laboratory consists of a number of devices each of which are equipped with sensors, actuators, web camera and intermediate hardware for communication purposes. These devices can be remotely monitored and controlled over the Internet. It allows students to conduct a range of experiments in their own time from a remote location. Such remote laboratories can be made accessible around the clock and the world for researchers and students. This way they can test their knowledge by performing non-simulated experiments on the hardware.

Several models of remote laboratories have been reported in literature; for different subjects' remote labs [1-32]. In MIT (Massachusetts Institute of Technology) a project named iLabs was initiated. It gave birth to the idea of remote labs for different engineering disciplines. Unlike conventional laboratories, iLabs can be shared anywhere throughout the world. iLabs' vision is to share expensive equipment and educational materials associated with lab experiments within higher education and beyond [2,31]. Lab share, National Support for Laboratory Resource Sharing, is an Australian Government-funded project. Its objective is to build a national network of remotely shared, accessible laboratories. This makes a greater number of high-quality, laboratory-based educational experiments possible that will be available to university and high school students from anywhere in Australia to around the world [3]. University of Deusto, Northern Illinois, University of Missouri-Kansas City, RMIT University
Australia, Blekinge Institute of Technology and many other universities also have their own live remote labs [4$8,32]$.

There are several approaches that can be considered to implement remote labs. An embedded web server based approach in which the server acts as a gateway between the external equipment and the computer system runs user interfaces which are built for interaction with the equipment [9-12]. Using Lab VIEW as controlling and communication software with the external devices, many remote labs have been developed [10-19]. SCADA (Supervisory Control and Data) also been used for remote monitoring and controlling of devices in remote labs [1922]. Some open-source software architectures and platforms are designed for E-learning and online laboratories like Moodel, SAHARA Labs and iLabs shared architecture [23-25].

Cloud computing is a new paradigm in making remote laboratories. Using cloud infrastructure, it is possible to pool all available resources together and provide a unified central storage for increased efficiency and feasibility [26].

IoT refers to an evocative development in the functions of the Internet. Even more than among people, it now enables communication among physical objects. It enables PPI (People-People Interaction), HMI (HumanMachine Interaction) and MMI (MachineMachine Interaction). The convergence of wireless technologies has evolved the concept of IoT to a greater extent. IoT, the next generation of Internet, is thought to revolutionize our whole lifestyle by interconnecting various "things". This includes computers, sensors, actuators, home appliances and other items to a global system using an IP (Internet Protocol) [27-30]. 
The proposed methodology for the remote lab is a combination of the above two paradigms; cloud computing and IoT. This lab is made for accessing FPGA boards remotely. External devices are interfaced through the LPT (Logical Parallel) Ports and COM (Communication) Ports. A data Acquisition and Control System is designed which acts as gateway between server and external devices. Support for multiple users accessing the lab simultaneously can be accommodated by an increase in the number of servers and devices. The combination of IoT with Cloud Computing provides new infrastructure that can support the proposed approach in many aspects. This includes monitoring and managing data/resources over the cloud, providing effective communication for serving the purpose of remote laboratories.

This paper has been divided into two sections; the first section describes the architecture design of the CLP and the second section elaborates on the implementation of the system.

\section{ARCHITECTURE DESIGN}

Fig. 1 describes the basic architecture of CLP. A modular approach is followed in the architecture design of CLP. The existing remote lab architecture can be enhanced by cloud services. The design is suited for local and remote network access. The data acquisition control systems are connected to their respective FPGA boards and both of these are connected to the servers. To avoid unauthorized usage and prevent equipment damage, security measures and locks are implemented.

Local users can access the labs using LAN (Local Area Network). The cloud represents the pool of resources. It keeps track of which boards and servers are in use as well as information of end users. Time scheduling, security, authentication and centralized storage for user and system data will be managed by cloud services. Real time video streaming is also enabled using webcam and HTTP (Hypertext Transfer Protocol). This allows users to see a live feed of the FPGA boards. The I/O (Input/Output) controls, bit stream files uploads and live transmission are all sub-modules of the developed system.

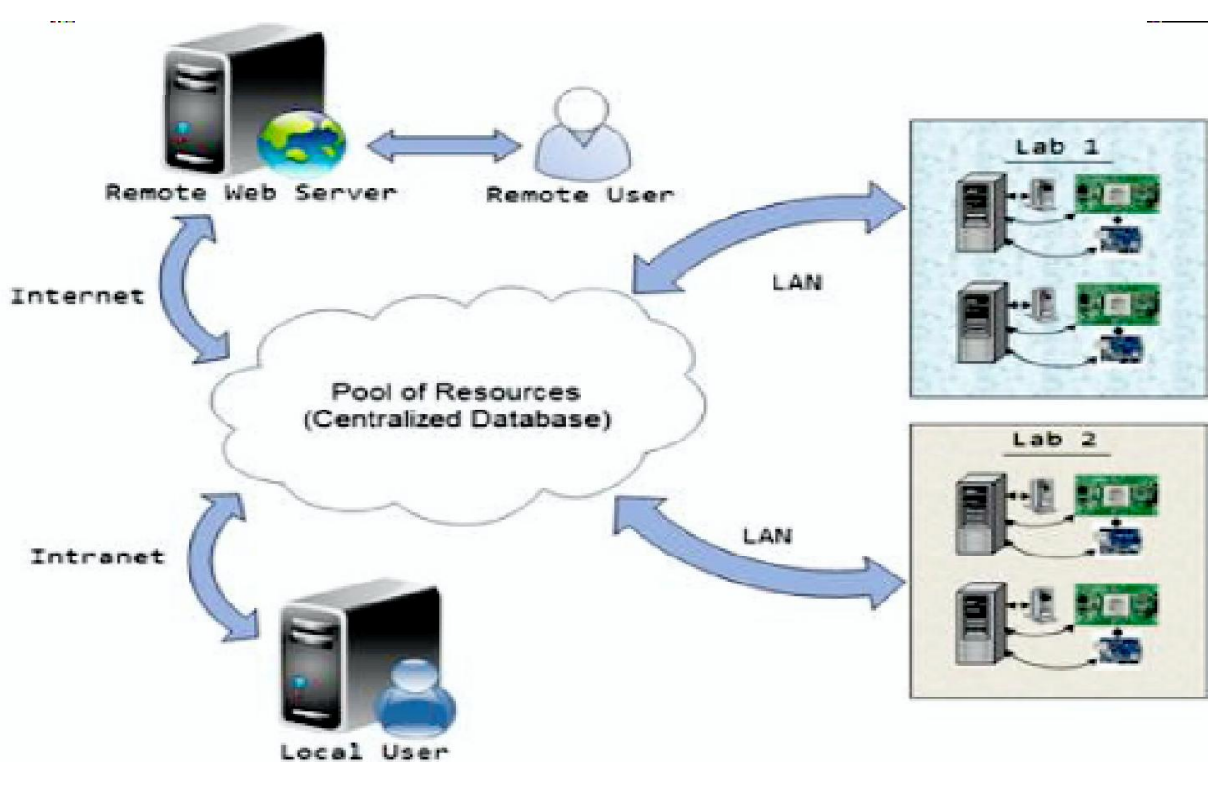

FIG. 1. ARCHITECTURE OF CLP 


\section{DESIGN IMPLEMENTATION OF CLP}

This section consists of three parts explaining the implementation of the hardware design and FPGA board interfacing, web interface design and cloud computing portions in that specific order.

\subsection{Hardware Design and FPGA Board Interfacing}

When interfacing any external hardware with a computer, its specifications need to be explored carefully. The boards which were selected are manufactured by XESS with Xilinx FPGA's Spartan-II and Spartan-III. Programming a board requires bit stream files which are created each time a program is generated in the Xilinx IDE (Integrated Development Environment). There are two options for uploading bit stream files: with Xilinx software or with the XESS custom software with GXLOAD.
The board is programmed by JTAG (Joint Test Action Group) interface in boundary scan mode through an LPT port. The bit stream file can be uploaded into the FPGA using GXLOAD or with iMPACT Xilinx tool. iMPACT is more feasible because it provides a command line interface.

The FPGA boards operate at low voltages. For the I/O channels, logic 1 is represented by $3.3 \mathrm{~V}$ and logic 0 by $0 \mathrm{~V}$. Assigning pins as mentioned in XESS manual allows us to operate inputs with switches and push buttons and display results on seven segment display or on bar LEDS.

In order to provide this functionality in the web interface, a system is required that acts as middleware between the computer and the FPGA boards. "The Data Acquisition and Control System" must have the capability of translating messages from user interface and sending relevant control signals to the FPGA board. It also has to be fault tolerant (Fig. 2).

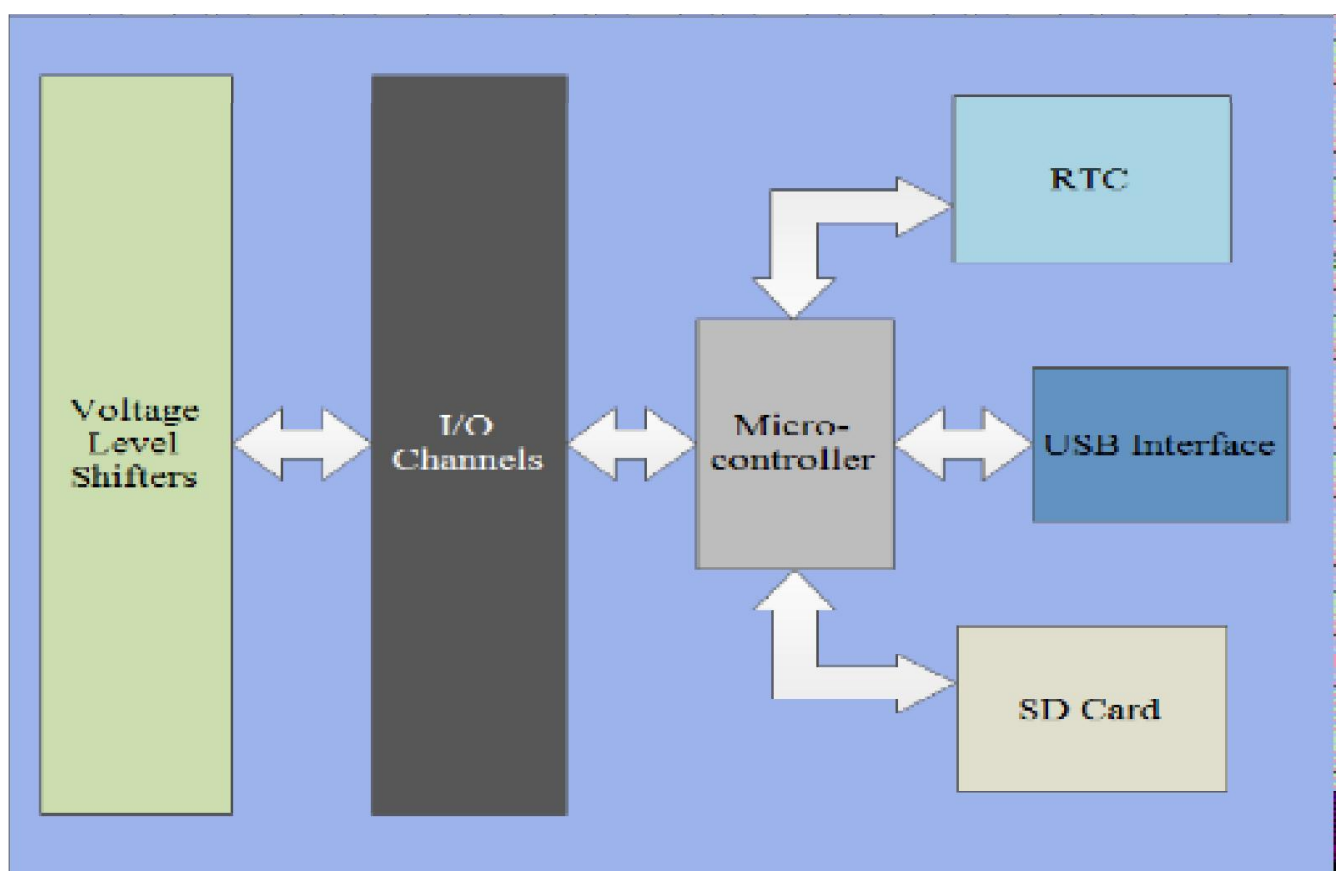

FIG. 2. STRUCTURE OF DATA ACQUISITION SYSTEM

Mehran University Research Journal of Engineering \& Technology, Volume 37, No. 4, October, 2018 [p-ISSN: 0254-7821, e-ISSN: 2413-7219] 
The system hardware consists of a microcontroller, an SD card, an RTC (Real Time Clock), and a transistor-based $\mathrm{I} / \mathrm{O}$ line controlling circuitry with a protection circuit as well.

This system is attached with a server using its COM port. The microcontroller receives messages from the user interface via the port, processes them and sends signals to the transistor-based I/O controlling circuitry which is connected to the FPGA pins. A real time clock is needed to store the time at which particular messages are received, usage history and pre-defined input patterns that can be generated when the user presses a command button from user interface. A camera is attached with the server by COM port. It is mounted over the FPGA board to show real-time status of the system when any particular input sequence applied.

\subsection{Web Interface}

The web interface has also been developed in a modular manner. Fig. 3 represents the structure of web application.
It consists of following modules:

- Serial communication

- $\quad$ FPGA board programing

- User authentication and registration

- $\quad$ Bit stream file storage

- $\quad$ Video streaming

- $\quad$ Mode selection and scheduling

- $\quad$ Device status and usage history

A web interface is provided to the user as a front end. Through this interface, a particular user can login and perform experiments from anywhere. The interface also provides options for comments and feedback.

User authentication and registration module is responsible for managing the users of the system. All the user specific functions such as registration and provision

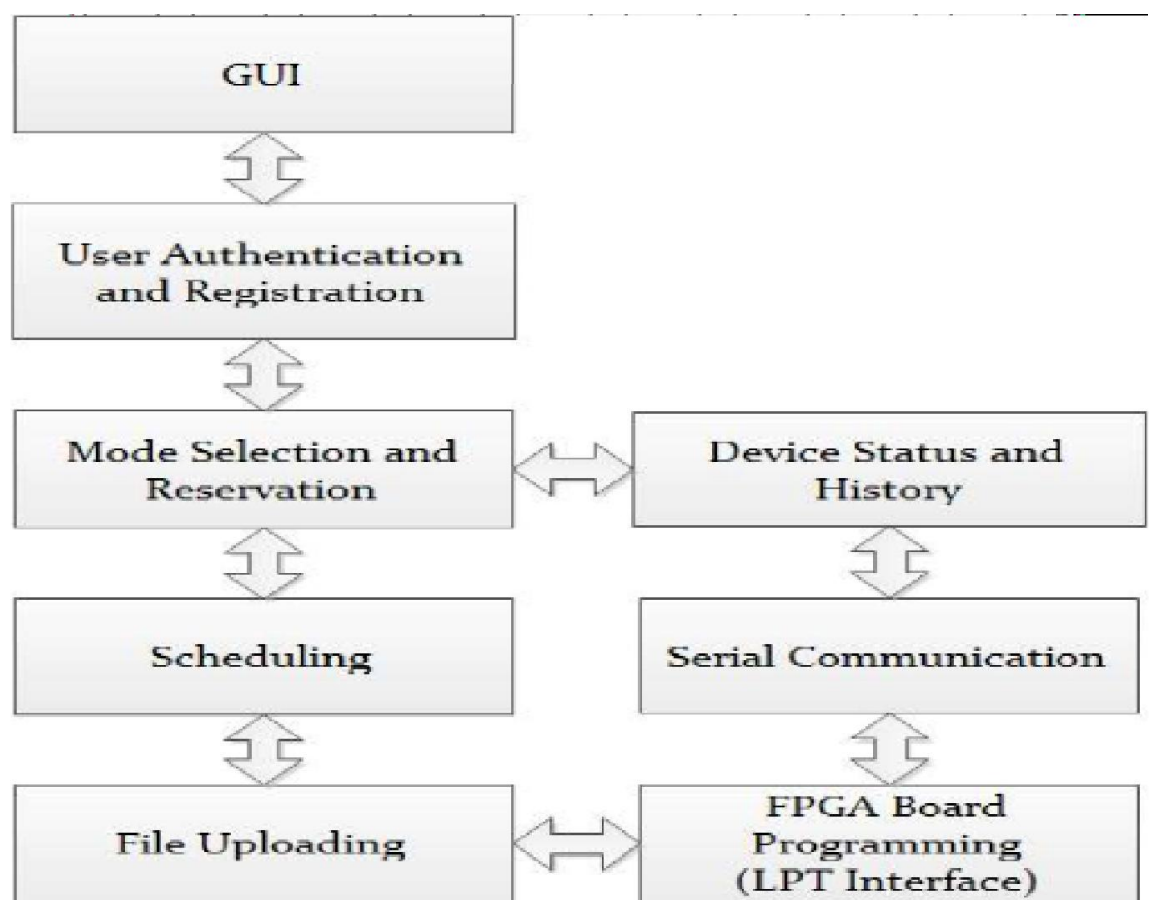

FIG. 3. WEB INTERFACE 
of ID and passwords were handled by this module. All of this information was saved in a centralized database which was developed using MySQL as shown in Fig. 4. There were 10 tables, the video stream and bitstream files that were directly saved in server using PHP (Hypertext PreProcessor) script. Their paths were saved in database for playing and record purposes.

The serial communication module is designed to communicate with the data acquisition and control system. The PHP filenamed "DIO.DLL" is designed for serial communication. The "non-thread save" version of the DLLfile was used.A separate PHP script is written for this task and called by main UI (User Interface) when needed. All the messages that were received and transmitted at any particular time were also saved in the database with each specific user.

FPGA board programming layer manages the uploading and removal of bit streams from the board. The PHP script written for this module invokes the iMPACT tool in batch mode with the user-provided bit stream file as an argument. After uploading, it shows the status of the board and the errors if any were encountered during the process.

The storage module stores the bit stream files in a database. It also checks whether the bit stream is appropriate for the board or not. The status of the device is shown to user.

Video streaming is enabled using VLC (Video LAN Client) media player's support for HTP. VLC is opensource software and provides the latest H.265/H.264 transcoding based video streaming over HTTP protocol. A PHP script is written for this module that establishes a connection with the port over which VLC is set to transmit the video. There is no noticeable latency problem in the transmission.

The recorded video files were also saved in a database to keep track of board usage and performance evaluation.

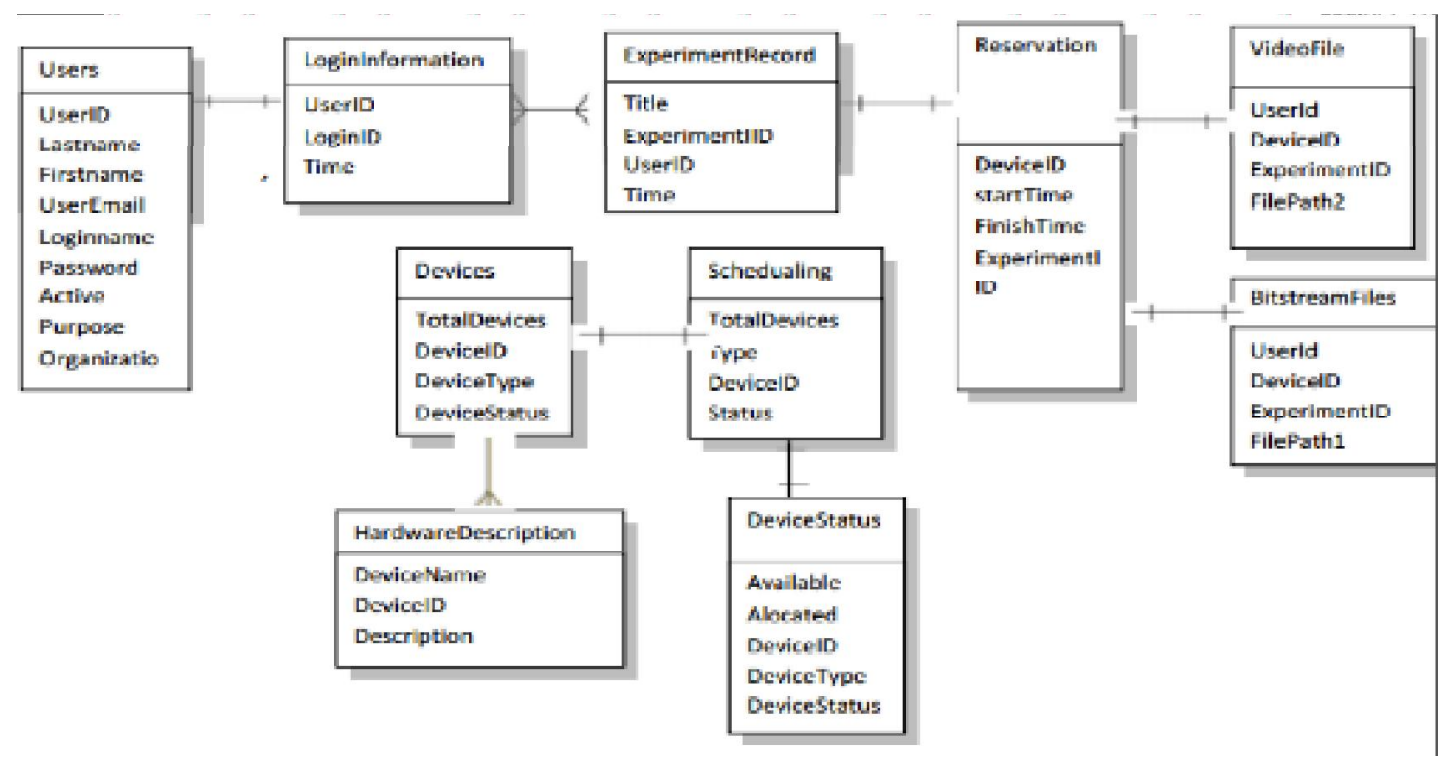

FIG. 4. DATABASE SCHEMA 
There are two types of modes available for using the FPGA boards. One is interactive mode and the second is offline mode. In interactive mode the user can reserve a board for some time and use it via a web interface. In the second mode the user doesn't need to interact directly with the board. The results of the experiment, after it has been performed automatically, are delivered to user. Mode selection is done by the user. If there are more users than the number of available boards, scheduling is done by the mode selection and scheduling script. If the time allotted to a particular user expires, then the intermediate results will be saved in a database and the user will be informed regarding the progress. Device status (idle or busy) is also saved in the database which is used by the mode selection and scheduling layer for checking whether a device is in use or if it can be provided to another user. All above module functionality are developed in separate scripts and called in the maininterface.

\section{CLOUD INFRASTRUCTURE}

Every time a user logs in or wants to use a system, any free device should be allocated to them irrespective of which server or device they worked on before. To enable this, we need a centralized infrastructure.

There are two possible approaches to establishing such a system. One is grid computing and the other is cloud computing. Cloud computing is best suited for these kinds of tasks.

The advantages of cloud computing are numerous.

- $\quad$ Cost effectiveness

- $\quad$ Device and Location Independency

- $\quad$ Reliable

- $\quad$ Secure
- Sustainable

- User-friendly

- Internet-centred

- $\quad$ Scalable and much more

The services involved in cloud infrastructure are IaaP (Infrastructure as a Protocol), SaaS (Software as a Service), and PaaS (Platform as a Service). The proposed CLP uses PaaS design. In this case, all servers will be updated automatically through a deployment process and resources can be shared seamlessly. This includes storage space as well as the FPGA boards. Cloud computing has enhanced the application of hardware and software and has also changed the application model. In our particular case different physical devices/equipment exist at different places that are connected to several servers. This setup was developed in a department using open-source Ubuntu open-stack. This cloud infrastructure was implemented on cluster nodes using open stack and this application is platform as service for equipment based labs. The reason behind this implementation is that the service providers just need to change front-end and attached device. In futures, this research will also include implementation using an open source cloud based API (Application Programming Interface).

\section{CASE STUDY}

The case study presented consists of different basic experiments of logic design like half-adder, full adder, multiplexer, logic gates, decoder, up/down counter and digital clock. They are presented in the web interface's tutorial section for the users as well. The FPGA boards are Xilinx Spartan-II and Spartan-III. PHP scripts were implemented for each event. The user interface of the lab is created using HTML, CSS as shown in Fig. 3 and Fig. 5. The interface contains following methods: 
(1) Get-Name of-Device (ID): Gives the name of the device.

(2) Reserved (ID): This reserves the board for a specified time period.

(3) Test-Board (ID): This method checks if the board is working properly

(4) Upload-Bit Stream (ID): In order to program the board, it needs a bit stream file.This method stores thebit file in the database.

(5) Program-Board (ID, BITSTREAM): This method burns the bit stream into the on-board flash memory.

(6) IS-FREE (ID): This method checks whether a particular device is free or not.
(7) Release (ID): This method is called automatically when the allotted time period expires or if users want to finish sessions themselves.

(8) Reset (ID): This method turns off the board, either as a command from the user.

\section{CONCLUSIONS}

Remote access provided through On-CLP facilitates students to access the laboratory even during the university after hours. This is very advantageous for the students from a learning point of view.

This paper has highlighted an idea to develop remotely accessible laboratories for all subjects, especially those which require costly and rare equipment. It can be scalable up to university level in Pakistan like other international universities did.

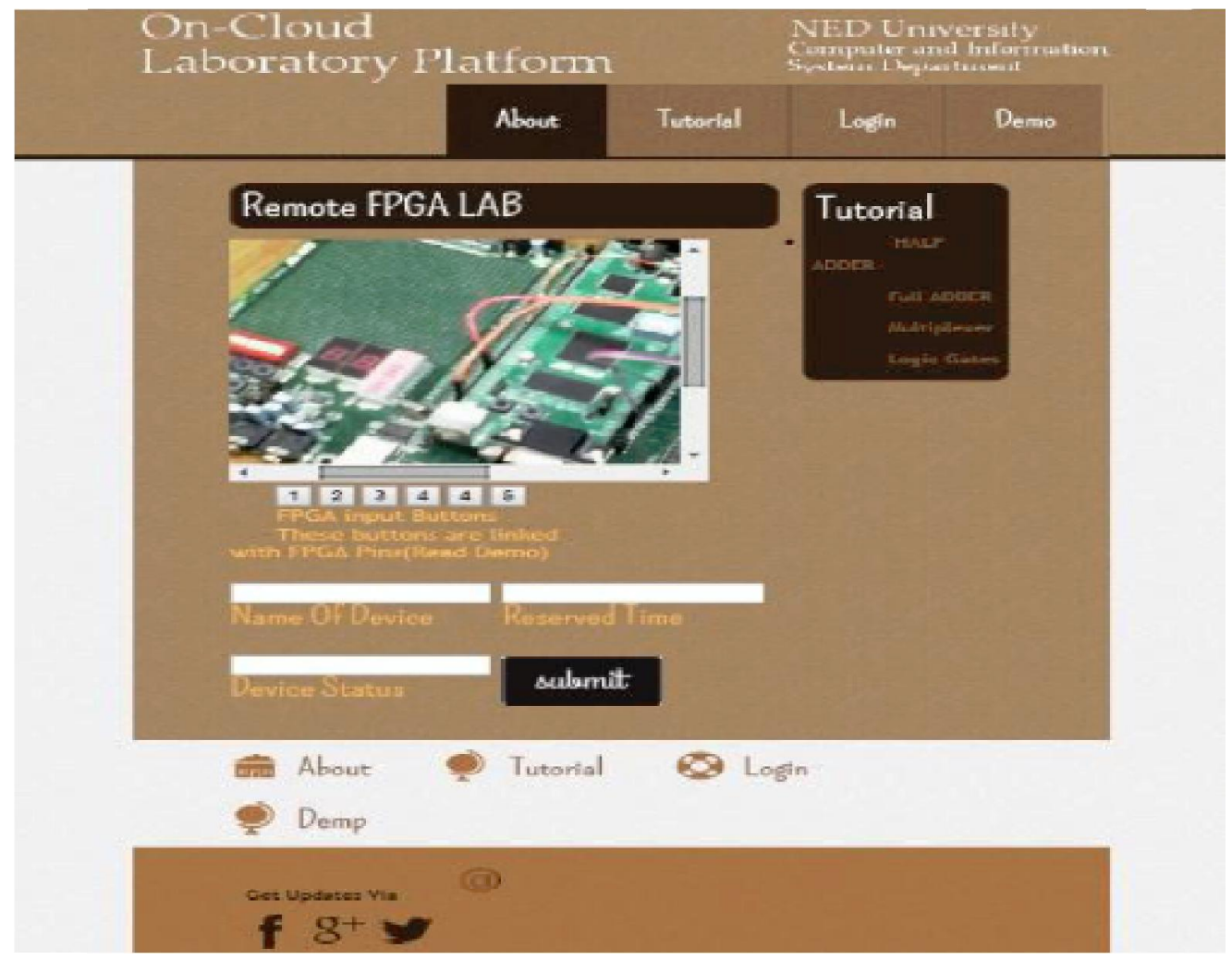

FIG. 5. WEB INTERFACE

Mehran University Research Journal of Engineering \& Technology, Volume 37, No. 4, October, 2018 [p-ISSN: 0254-7821, e-ISSN: 2413-7219] 
The current technologies for implementing remote laboratories, their relation to the learning process and their evolution in light of future developments in IoT have been discussed in this paper. The project described and all the development tools used to create it are open source and free-ware.

\section{ACKNOWLEDGEMENT}

Authors would like to acknowledge NED University of Engineering \& Technology, Karachi, Pakistan, to provide resources and facilities for the research conducted in this study.

\section{REFERENCES}

[1] Alves, G.R., Fidalgo, A., Marques, A., Viegas, C., Felgueiras, M.C., Costa, R., Lima, N., Castro, M., Díaz-Orueta, G., and Ruiz, E.S.C., "Spreading Remote Lab Usage a SystemA Community-A Federation", IEEE 2nd International Conference on Portuguese Society for Engineering Education, Portugals, 2016.

[2] Harward, V.J., Del Alamo, J.A., Lerman, S.R., Bailey, P.H., Carpenter, J., DeLong, K., Felknor, C., Hardison, J., Harrison, B., and Jabbour, I., "The iLab Shared Architecture: A Web Services Infrastructure to Build Communities of Internet Accessible Laboratories", Proceedings of IEEE, Volume 96, No. 6, pp. 931-950, 2008.

[3] Labshare, "Lab Share Project", Website:http:// www.labshare.edu.au/project/, (Access Date: $16^{\text {th }}$ March, 2016).

[4] "WebLab-Duesto", Website: http://weblab.deusto.es/ website/, (Access Date: 17 $7^{\text {th }}$ April, 2016).

[5] Orduna, P., Rodriguez-Gil, L., Angulo, I., Dziabenko, O., Hernandez, U., Lopez-de-Ipina, D., and Garcia-Zubia, J., "Towards a MicroRLMS Approach for Shared Development of Remote Laboratories", 11 th International Conference on Remote Engineering and Virtual Instrumentation, February, 2014.

[6] "NIU Internet Accessible Remote Lab Project", Website: http://www.niu.edu/remotelab/, (Access Date: $16^{\text {th }}$ March, 2016).
[7] UMKC Remote Labs Project Website: http:// www.umkc.edu/is/remotelabs/, (Access Date: $6^{\text {th }}$ April, 2016).

[8] “MIT Remote Labs", Website: http://www.rmit.edu.au/ teaching/technology/remotelabs, (Access Date: $16^{\text {th }}$ March, 2016).

[9] Gustavsson, I., "User-Defined Electrical Experiments in a Remote Laboratory", Proceedings of American Society for Engineering Education Annual Conference \& Exposition, 2003.

[10] Yang, L., Jiang, L., Yue, K., and Pang, H., "Design and Implementation of the Lab Remote Monitoring System Based on Embedded Web Technology", International Forum on Information Technology and Applications, Volume 2, pp. 172-175, 2010.

[11] Otoakhia, E., Jenmanachaiyakun, T., Afaneh, A., Alzebda, S., Mani, M., Sonbul, O., and Kalashnikov, A.N., "Embedded Web Server for Remote Laboratory Access Founder Graduate Students Studying Electronic Engineering", IEEE International Symposium on Circuits and Systems, 2011.

[12] Yudil.F., Sudibyo, H., Putri, R.A.A., and Arifin, A.S., "The Design of Embedded Web Server for Remote Laboratories Microcontroller Systems Experiment", TENCON IEEE Region 10 Conference, 2011.

[13] YudiL.F., Putri, R.A.A., and Sudibyo, H., "Remote Laboratories Multiuser based on Embedded Web Server", 9th International IEEE Conference on Remote Engineering and Virtual Instrumentation, 2012.

[14] Gallardo, S., Barrero, F., Toral, S.L., and Durán, M.J., "eDSPlab: A Remote-Accessed Instrumentation Laboratory for Digital Signal Processors Training Based on the Internet", IEEE 32nd Annual Conference on Industrial Electronics, 2006.

[15] Hercog, D., Gergi, B., Uran,S., and Jezernik, K., “A DSPBased Remote Control Laboratory", IEEE Transactions on Industrial Electronics, Volume 54, No. 6, December, 2007. 
[16] Mallik, A., and Gupta, S.D., "Modelling of MEMS Based Temperature Sensor and Temperature Control in a Petrochemical Industry Using LabVIEW", International Conference on Computer and Automation Engineering, 2009.

[17] Straatsma, M., Cox, D., Ctistis, C., and Bartz, R., "Development and Enhancement of RLab A Remote Laboratory System", $4^{\text {th }}$ International Conference on Systems and Networks Communications, 2009.

[18] Pengfei, L., and Luhua, L.N., "Remote Control Laboratory Based On LabVIEW", 2 ${ }^{\text {nd }}$ International Conference on Intelligent Computation Technology and Automation, 2009.

[19] Yazidi, A., Henao, H., Capolino, G., Betin, F., and Filippetti, F., “A Web-Based Remote Laboratory for Monitoring and Diagnosis of AC Electrical Machines”, IEEE Transactions on Industrial Electronics, Volume 58, No. 10, October, 2011.

[20] Orduña, P., García, Z.J., Rodriguez, G.L., Irurzun, J., López-de-Ipiña, D., and Gazzola, F., "Using LabVIEW Remote Panel in Remote Laboratories: Advantages and Disadvantages", IEEE Global Engineering Education Conference, 2012.

[21] Shankar, B., Sarithlal, M.K., Sharat, S., Freeman, J., and Achuthan, K., "Remote Triggered Virtual Laboratory for Hooke's Law Using LabVIEW”, 39th Annual Conference of Industrial Electronics Society, 2013.

[22] Titov, I., and Titov, E., "Labicom.net - Putting Your Laboratory Online in Less than Five Minutes with Web Pager Tool Automatic Generation and Real-Time Control of a LabVIEW Based Laboratory Server from Plug in Less HTML Page”, 2nd Experiment@International Conference, 2013.

[23] Salihbegovic, A., and Tanovic, O., "Internet Based Laboratories in Engineering Education", 30th International Conference on Information Technology Interfaces, 2008.
[24] Aydogmus, Z., and Aydogmus, O., "A Web-Based Remote Access Laboratory Using SCADA”, IEEE Transactions on Education, Volume 52, No. 1, February, 2009.

[25] Nedic, Z., and Nafalski, A., "Suitability of SCADA for Development of Remote Laboratories", 10th International Conference on Remote Engineering and Virtual Instrumentation, 2013.

[26] Tawfik, M., Cristóbal, E.S., Pesquera, A., Gil, R., Martin, S., Diaz, G., Peire, J., Castro, M., Pastor, R., and Ros, S., "Shareable Educational Architectures for Remote Laboratories", Conference on Technologies Applied to Electronics Teaching, 2012.

[27] Correia, R.C., Fonseca, J.M., and Donellan, A., "Euronet Lab a Cloud Based Laboratory Environment", IEEE Global Engineering Education Conference, 2012.

[28] Latorre, G.M., Fernandez, G., Ruiz, E., Martín, A.P., and Castro, G.M., "Rethinking Remote Laboratories: Widgets and Smart Devices", IEEE Frontiers in Education Conference, 2013.

[29] Anderson, J., and Rainie, L., "The Internet of Things will Thrive by 2025", Pew Research Center, May 14 2014.

[30] Vermesan, O., and Friess, F., "Internet of Things Converging Technologies for Smart Environment and Integrated Ecosystems", Denmark River Publishers, Chapter-6, pp. 245-257, Alborg, Denmark, 2013.

[31] MIT iLabsProject Website: http://ilab.mit.edu/wiki/, (Access Date: 15 $5^{\text {th }}$ March, 2016).

[32] Milan, S., Nikola, D., Nedeljkoviæ, M.S., and Èantrak,D.S., "Remote Labs and Problem Oriented Engineering Education”, IEEE Global Engineering Education Conference, 2017. 